\title{
El Diamante Competitivo. Una propuesta para el cluster de las microempresas del sector de la confección de la parroquia Pasa en Tungurahua
}

URL: http://revistas.uta.edu.ec/erevista/index.php/bcoyu/article/view/721 Dol: http://dx.doi.org/10.31164/bcoyu.22.2019.721

Nelson Lascano - Aimacaña ${ }^{1}$; Carla Pérez -Lara ${ }^{2}$

Fecha de recepción: 15 de noviembre de 2016

Fecha de aceptación: 9 de septiembre de 2019

\section{Resumen}

El presente trabajo investigativo tiene como propósito establecer una propuesta para potenciar el crecimiento y posicionamiento de las microempresas del sector de la confección de la Parroquia Pasa en Tungurahua. La investigación tiene dos fases: la que analiza los sistemas de comercialización y la rentabilidad; y la otra fase orientada a establecer el Diamante Competitivo, mediante un Diagnóstico de los encadenamientos productivos y el establecimiento de estrategias competitivas para este sector. Mediante el desarrollo del modelo de cluster como propuesta de investigación, se estipula las condiciones adecuadas para su desarrollo y crecimiento empresarial de este sector.

Palabras clave: Microempresas, sistemas de comercialización, rentabilidad, cluster, estrategia competitiva.

\section{Abstract}

This research work aims to establish a proposal for Growth and positioning of Pasa's micro-enterprises in the clothing industry in Tungurahua. The research has two phases: first, analyze marketing systems and profitability; and the other phase oriented at establishing the Competitive Diamond, through a diagnosis of productive linkages and establishing competitive strategies for this sector. Through the development of cluster model as a research proposal, stipulated that has the appropriate conditions for their development and business growth

Keywords: Microenterprises, marketing, profitability, cluster, competitive strategy.

\section{Introducción}

Esta investigación referencia a las microempresas del sector de la confección de Pasa provincia de Tungurahua, por ser uno de los sectores económicos más importantes de la parroquia y cuenta con un potencial de crecimiento. Sin embargo, se ha determinado que carecen de eficientes sistemas de comercialización de sus productos lo cual ha repercutido en los niveles de rentabilidad. Con estos antecedentes, se diseñó el diamante competitivo para el cluster de las microempresas del sector de la confección de la Parroquia Pasa en Tungurahua para potenciar el crecimiento y posicionamiento del sector.

Adicionalmente, es necesario comprobar la relación estadística entre los Sistemas de Comercialización y la Rentabilidad del cluster de las microempresas, para el establecimiento de planes de acción. Así también, se requiere, establecer las estrategias competitivas que permitan el logro del desarrollo económico local y sustentable de las microempresas mediante el diagnóstico, de los encadenamientos productivos o clusters para conocer su importancia en el sector.

\section{Metodología}

La investigación es no experimental, documental, de campo, y con un enfoque de tipo cuantitativo y cualitativo, inicialmente se realizó el estudio de las variables Sistemas de Comercialización y Rentabilidad, las cuales constituyeron eslabones determinantes en el establecimiento de soluciones oportunas que faciliten el crecimiento del sector. Es importante mencionar que los instrumentos utilizados para la recolección de información fueron la encuesta y la ficha de observación.

Los cuestionarios fueron sometidos al test de confiabilidad y validez "Alfa de Cronbach" para medir la fiabilidad de los mismos. Con un resultado de 0.86 se puede concluir que la encuesta aplicada cuenta con una fiabilidad de escala de medida alta. Por otro lado, la población está constituida por 10 microempresarios (representativos) quienes elaboran y fabrican camisas y demás prendas de vestir.

Los niveles de rentabilidad de las microempresas se determinaron a través del indicador ROE, que establece el crecimiento o decrecimiento de la rentabilidad de las microempresas durante el último año. Así también, el estadígrafo seleccionado para la comprobación de la hipótesis planteada fue la prueba de Correlación, que determina el nivel de impacto entre las variables.

Para verificar la hipótesis, se utilizó el método t de student. Para la cuantificación de la variable independiente los sistemas de comercialización se la representarán mediante las ventas; pues son variables que intervienen en la comercialización y se encuentran mutuamente relacionadas. Por tanto, las variables que intervienen en el análisis de correlación son el porcentaje de incremento o disminución de las ventas y el porcentaje de rentabilidad del año 2015; tal como se observa en la Tabla 1.

\begin{tabular}{rrrrrr}
\multicolumn{6}{c}{ Tabla 1. Cálculo de r } \\
\cline { 2 - 6 } & Ventas & $\begin{array}{c}\text { Renta- } \\
\text { bilidad }\end{array}$ & V * R Ventas $^{2}$ & $\begin{array}{c}\text { Renta- } \\
\text { bilidad }^{2}\end{array}$ \\
1 & $\mathbf{0 , 2 5}$ & 0,375 & 0,094 & 0,063 & 0,141 \\
2 & $\mathbf{0 , 6 0}$ & 0,778 & 0,467 & 0,360 & 0,605 \\
3 & 0,20 & 0,333 & 0,067 & 0,040 & 0,111 \\
4 & $\mathbf{0 , 2 0}$ & 0,125 & 0,025 & 0,040 & 0,016 \\
5 & 0,30 & 0,167 & 0,050 & 0,090 & 0,028 \\
6 & 0,50 & 0,667 & 0,333 & 0,250 & 0,444 \\
7 & 0,30 & 0,667 & 0,200 & 0,090 & 0,444 \\
8 & 0,50 & 0,500 & 0,250 & 0,250 & 0,250 \\
9 & 0,35 & 0,357 & 0,125 & 0,123 & 0,128 \\
10 & 0,50 & 0,455 & 0,227 & 0,250 & 0,207
\end{tabular}

$$
\mathrm{r}=\frac{n \sum x y-\sum x \sum y}{\sqrt{n \sum x^{2}-\left(\sum x\right)^{2} \sqrt{n \sum y^{2}-\left(\sum y\right)^{2}}}} \quad \mathrm{r}=0.72262389
$$

Elaboración propia a partir de ficha de observación (2015)

El coeficiente de correlación de 0.72 muestra que existe una correlación alta entre las variables relacionadas. Para la determinación t student se utiliza la siguiente fórmula.

$$
\mathrm{t}_{\mathrm{c}=}=\frac{\mathrm{r} \sqrt{\mathrm{n}-2}}{\sqrt{1-\mathrm{r}^{2}}} \quad \mathrm{t}_{\mathrm{c}}=2.956838165
$$

Considerando el valor de t tabular de 1.8595 y la presente regla de decisión "Acepto $\mathrm{H}_{0}$ Sí $\mathrm{t}_{\mathrm{c}}<\mathrm{t}_{\mathrm{t}}$ " se llega a las siguientes conclusiones.

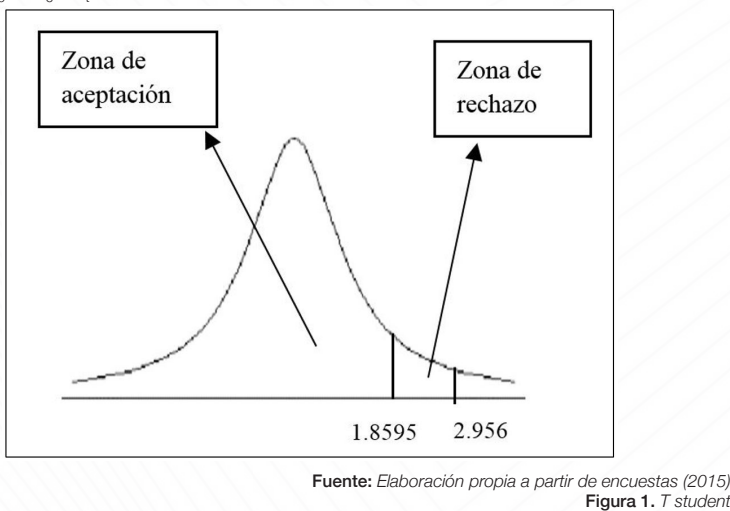

Como el valor de t calculado ( ) es mayor a 1,86 (tt) con 8 grados de libertad y un a de 0.05 , se RECHAZA la Hipótesis Nula y se ACEPTA la Alterna; es decir, "Si hay diferencia estadística significativa", pues los Sistemas de Comercialización sí tienen un impacto en la Rentabilidad de las microempresas del sector de la confección de la Parroquia Pasa; tal como se detalla en la Figura1.

\section{Resultados}

La promoción y publicidad constituyen variables determinantes de la comercialización, ya que permiten posicionar el producto en la mente del consumidor y por ende el incremento del nivel de ventas. Sin embargo, la mayor parte de las microempresas del sector motivo de estudio no aplican promoción y publicidad; es por esto, que el producto no es muy reconocido ni pretendido en el mercado.

Los productores del sector de la confección han logrado distribuir sus productos en el mercado local y regional de la mejor manera posible utilizando intermediarios que han facilitado la distribución del producto en los diferentes segmentos de mercado; sin embargo, aún no lo hacen de una manera técnica y adecuada.

Se determinó que casi siempre llegan los productos a los clientes finales y se tiene contacto directo con los mismos; pues, algunas de las microempresas elaboran los productos y son ellas mismas las encargadas de distribuirlos y venderlos directamente al consumidor.

Se asevera además, que de una u otra manera se trabaja con varios niveles de intermediarios para la comercialización del producto; pues, son los encargados 
de colocarlos en varias ciudades del país. Cabe recalcar, que pocas veces se tiene control sobre ellos, una vez que son entregados; ya que son los productores quienes deciden sobre las condiciones de venta.

En el caso del resultado ROE favorable en promedio de las microempresas es de 0.44 , lo cual indica la utilidad disponible para los accionistas/propietarios o utilidad neta financiera y significa un rendimiento de piezas sobre la inversión realizada por los accionistas al inicio del periodo, representada ésta por el patrimonio. Efectivamente, se determinó que los niveles de rentabilidad en la mayor parte de las microempresas se han incrementado durante el último año, claro que no al nivel de años anteriores.

\section{Etapas del Modelo Operativo}

En esta etapa se aborda la propuesta o vía de solución para mejorar el posicionamiento de las microempresas en el mercado desarrollando un modelo de diamante competitivo para el cluster de las mismas mediante el establecimiento de una estrategia competitiva. Esta etapa se desarrolla en tres fases descritas a continuación.

Etapa1. Diagnóstico de los encadenamientos productivos.

Cadena de valor. "La cadena de valor contiene el valor total y consta de actividades relacionadas con valores de margen. Se trata de actividades físicas y tecnológicamente específicas que se llevan a cabo. Son las estructuras mediante las cuales se crea un producto para los compradores." (Porter, 2002) Es decir, muestra el conjunto de actividades y funciones ligadas que se llevan a cabo internamente en una organización. A la vez, permite determinar la ventaja competitiva que tiene la organización en relación a sus competidores y facilita la toma oportuna de decisiones de los representantes de las mismas.

Para diagnosticar la ventaja competitiva de las microempresas del sector de la confección de la Parroquia Pasa, es necesario definir su cadena de valor para que compitan en dicho sector económico conociendo así tanto sus eslabones fuertes como los débiles.

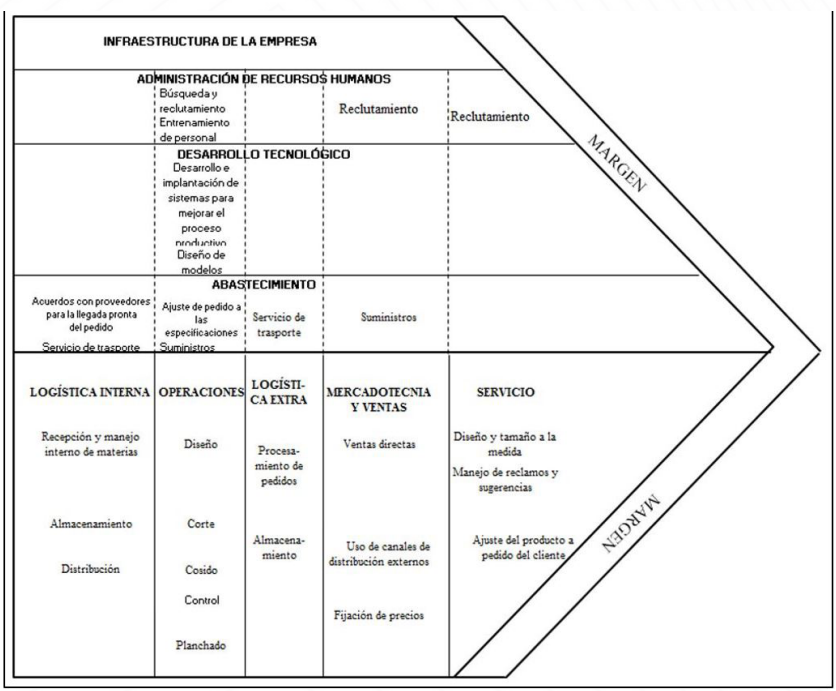

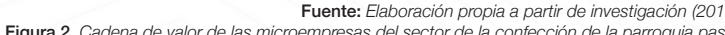

Se diagnosticó que la cadena de valor del cluster de las microempresas no se encuentra en las condiciones adecuadas que permita el crecimiento del sector pues algunos eslabones de las actividades primarias como la logística de entrada o servicio no son aplicados por las microempresa así como las actividades de apoyo. Sin embargo, cuentan con un alto potencial de crecimiento y progreso debido a que cuentan con actividades que las han desarrollado eficientemente. Así también, una vez que se potencie la cadena de valor se desarrollará un valor agregado al producto que favorecerá el desarrollo del sector; tal como se aprecian en la Figura 2.

Planificación estratégica. En el análisis FODA se puede determinar que los factores internos de las microempresas del sector de la confección no tienen resultados favorables que les permitan desarrollarse empresarialmente en la industria. Pues sus fortalezas son escasas y sus debilidades son mucho mayores, tal como se puede apreciar en la Tabla 2. No obstante, no se debe pasar por alto que las fortalezas alcanzadas por las microempresas deben ser reforzadas para alcanzar el potencial que requiere el cluster. Claro que, mediante este estudio, posteriormente, este sector realizará los respectivos planes estratégicos, de acción y agendas de control y evaluación para el cumplimiento de la planificación estratégica realizada inicialmente.

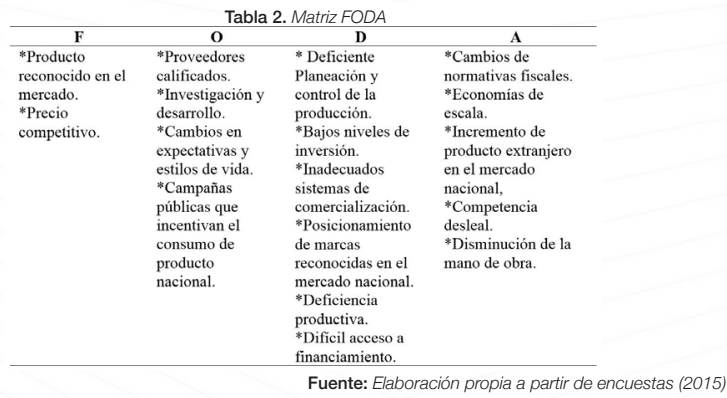

Capacidad de producción. Se conoce que la capacidad real de producción mensual por trabajador en cada microempresa varía de entre 500 a 800 unidades de producto, dependiendo exclusivamente del mes, el período y la demanda del mercado. Cada trabajador elabora aproximadamente 110 unidades diarias y 590 mensuales, tomando en cuenta horas extras y suplementarias. Las horas hombre al mes varían de 240 a 280 en la mayor parte de las microempresas. Es importante aclarar que las microempresas cumplen con las disposiciones del Código de Trabajo vigente, pues laboran 8 horas ordinarias, 3 horas suplementarias diarias y 5 horas extras a la semana (sábados); dando así un total de 240 horas mensuales. Sin embargo, éstas sobrepasan su estimación dependiendo del mes laborado y temporadas de alta demanda.

Etapa2. Análisis del Diamante de Porter. condiciones básicas para la formación de Cluster.

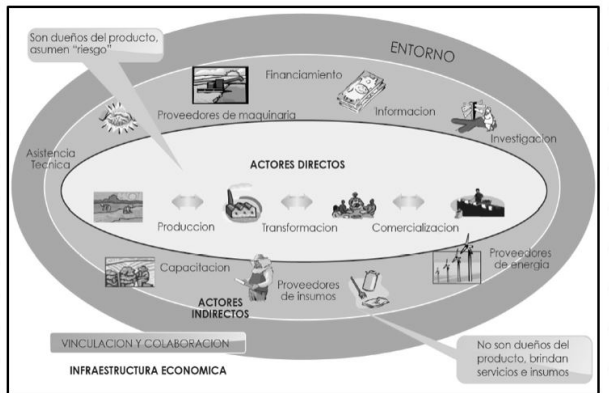

Fuente: Agenda de Productividad y Competitividad de Tungurahua, H. Gobierno Provincial de Tungurahua Figura 3. Actores de un Cluster

En esta etapa se diseña el diamante competitivo para el cluster de las microempresas del sector de la confección de la Parroquia Pasa. En este enfoque se destacan cuatro aspectos básicos en el clima competitivo y de desenvolvimiento de las microempresas, que al operar de forma simultánea en el tiempo y en el espacio crean las condiciones para la formación y el desarrollo del cluster.

Es importante recordar que un cluster

es un grupo geográficamente denso de empresas e instituciones conexas, pertenecientes a un campo concreto, unidas por rasgos comunes y complementarias entre sí. Los cúmulos adoptan varias formas, dependiendo de su profundidad y complejidad, pero la mayoría de ellos comprenden empresas de productos o servicios finales, proveedores de materiales, componentes, maquinaria y servicios especializados, instituciones financieras y empresas de sectores afines. En los cúmulos también suelen integrarse empresas que constituyan eslabones posteriores de la cadena. (Porter, 2003) Dicho concepto se puede apreciar en la Figura 3.

La Figura 4; de la misma manera muestra los componentes que integran el diamante competitivo, destacando cuatro aspectos básicos en el clima de negocios que determinan las ventajas competitivas de las empresas. Al operar de forma simultánea en el tiempo y en el espacio, estos aspectos crean las condiciones para la formación y el desarrollo de los clusters en determinados sectores y lugares.

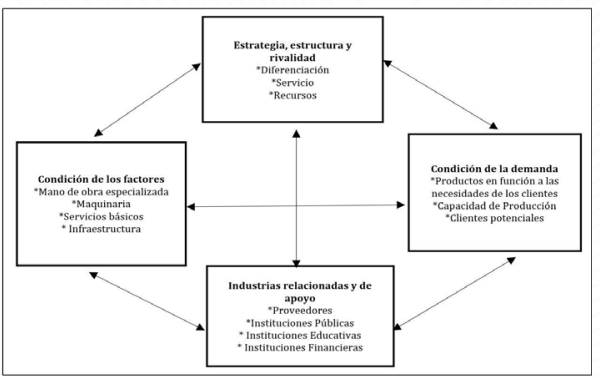

Fuente: Elaboración propia a partir de investigación (2015) Figura 4. Diamante de Porter 


\section{Condiciones de los Factores.}

Las microempresas del sector de la confección cuentan con las condiciones de los factores básicos como tierra, clima y servicios básicos. Así también, cuentan en su mayoría con los factores avanzados o especializados como maquinaria, mano de obra especializada, infraestructura, entre otros que han favorecido el desarrollo de la actividad productiva; sin embargo, aún necesitan mejorarlos en función a los requerimientos del mercado. Así como los establece (Garrido \& Pérez, 1998) que los factores de la producción son elementos bases que permiten crear competitividad empresarial.

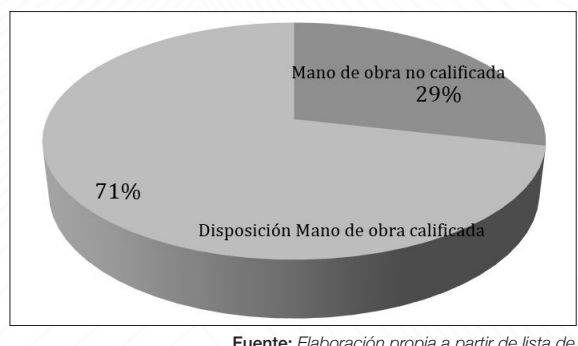

Fuente: Elaboración propia a partir de lista de verificación (2015) Figura 5. Factor de producción trabajo

\section{Condiciones de la Demanda}

La demanda de los productos del cluster de las microempresas del sector de la confección requiere de productos que principalmente cumplan con las características y requerimientos de los clientes. Es así, que el sector de la confección cuenta con una demanda altamente exigente, la misma que presionará para que las microempresas elaboren productos cada vez mejores y en función a sus necesidades. Por lo que uno de los factores de éxito en este sector productivo es el diseño de camisas especialmente.

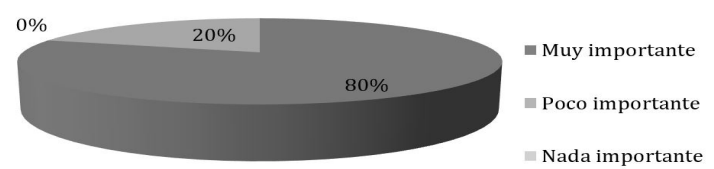

Fuente: Elaboración propia a partir de encuestas (2015) Figura 6. Importancia de la calidad para la demanda

Una clara muestra de la exigencia de la demanda se presenta en Fig. 6, donde la importancia de la calidad de la demanda, al ser un requisito inherente en los productos, los clientes consideraron de vital importancia (80\%) al momento de adquirir los productos de las microempresas motivo de estudio

\section{La Existencia de Industrias Relacionadas y de Apoyo.}

La presencia de instituciones e industrias que apoyen el desarrollo de la actividad productiva de un determinado sector económico se convierten en un estimulante para la creación de los cúmulos. El sector estudiado cuenta con la existencia de industrias relacionadas y de apoyo para el desarrollo del diamante competitivo del cluster de las microempresas como proveedores cercanos, instituciones públicas como el MIESS, MIPRO, los GADS entre otros; e instituciones educativas como universidades y colegios del sector; en un 80\%, tal como lo muestra la Fig. 7.

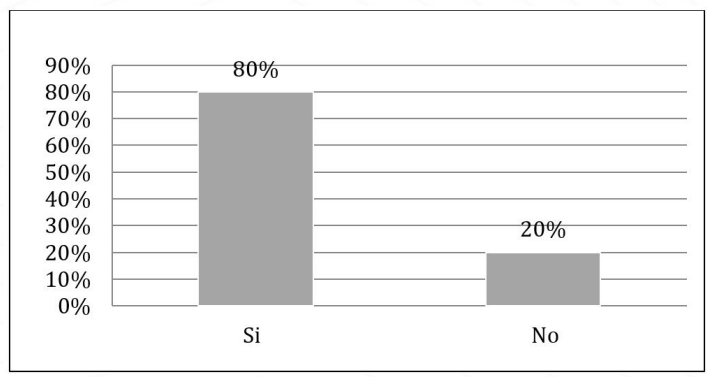

Fuente: Elaboración propia a partir de encuestas (2015) Figura 7. Presencia y apoyo de industrias e instituciones relacionadas

\section{Esquema de Estrategia y Rivalidad de la Industria}

Es necesaria una fuerte rivalidad local que garantice el fortalecimiento del cluster del sector de la confección. Por ello, se ha definido que existe un nivel relevante de competencia entre las microempresas pues tratan de diferenciase y distinguirse entre ellas, representado en un $70 \%$ mostrado en la Fig. 8. Es decir, cuentan con los recursos y condiciones que les permiten competir y diferenciarse del resto de microempresas. Sin embargo, el resto de ellas no han hecho mayor esfuerzo por crecer y distinguirse en el mercado, pues no disponen de los medios o recursos necesarios para realizarlo.

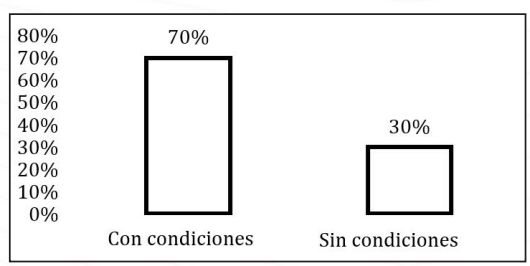

Fuente: Elaboración propia a partir de encuestas (2015) Figura 8. Nivel de rivalidad entre competidores del sector

Etapa 3. Establecimiento de estrategias competitivas.

Se determinó que en función de las condiciones del mercado y el resto de contextos que requiere el diamante competitivo, el cluster deberá aplicar la estrategia de diferenciación. La mayor parte de su demanda requiere un producto de alta calidad sin importar el precio que esto conlleve. Esta estrategia vendrá acompañada de la estrategia de enfoque, misma que orientará a los productores a su mercado meta en función del producto que ofertan.

\section{Conclusiones}

- Se determinó mediante la comprobación de la hipótesis que los Sistemas de Comercialización impactan significativamente en la Rentabilidad de las microempresas del sector de la confección de la Parroquia Pasa; por lo que se deben establecer de manera correcta los lineamientos de diseños y aplicación de los respectivos sistemas.

- Los Sistemas de Comercialización utilizados por los microempresarios del sector de la confección no han sido aplicados eficientemente acorde a las exigencias del mercado.

- Los niveles de Rentabilidad de las Microempresas del Sector de la Confección de la Parroquia Pasa se han incrementado en su mayoría; sin embargo, no como años atrás. Por tanto, dichos resultados no son óptimos para su crecimiento en el mercado.

- La estrategia genérica que permitirá el crecimiento y desarrollo de la ventaja competitiva del cluster del sector de la confección será la diferenciación, acompañada de la estrategia de enfoque.

\section{Referencias}

Capó, J., Expósito, M., \& Masiá, E. (2007). La importancia de los clusters para la competitividad de las PYME en la economía global. Eure, 119-133.

Cervilla de Olivieri, M. (2007). Estrategias para el desarrollo empresarial: Asociatividad en el sector plástico venezolano. Revista de Ciencias Sociales, 230 $-248$

Chase, R., Jacobs, R., \& Aquilano, N. (2009). Administración de la producción y operaciones (Duodécima ed.). Mexico, D.F.: McGraw-Hill/Interamericana de Editores, S.A. de C.V.

Córdova Padilla, M. (2012). Gestión Financiera. Bogotá D.C.: Ecoe Ediciones.

Corrales, S. (2007). Importancia del cluster en el desarrollo regional actual. Frontera norte, $173-201$

Gargante, T. (1992). Sector textil. La apuesta de la comercialización. Mercasa, 102-108

Garrido, M. J., \& Pérez, M. P. (1998). Sarisfacción de los clientes internos: EL Papel. Academia Europea de Dirección y Economía de la Empresa, AEDEM, 13-28.

Estolano, D., Castillo, I., Berumen, M., \& Mendoza, J. (2013). El escenario de competencia de la Industria Gastronómica de Cancún basado en las cinco fuerzas de Porter. El Periplo Sustentable, 67 - 97.

Guerrero García, M., \& Pérez Martínez, Y. (2009). Procedimiento para el proceso de comercialización en cadenas de tiendas. Edición electrónica gratuita.

H. Gobierno Provincial de Tungurahua. (2010). Agenda de Productividad y Competitividad de Tungurahua. Ambato: H. Gobierno Provincial de Tungurahua. Larrubia Vargas, R. (2010). Una visión desde el Sector Hortofrutícola. Boletín de la Asociación de Geógrafos Españoles, 117-141.

Muñoz, D. (2009). Administración de operaciones. México D.F.: Cengage Learning.

Parada Gómez, A. M. (2009). La fuerza motriz en el desarrollo económico de las Micros, Pequeñas y Medianas Empresas (Mipymes) en la Cadena Global de Ropa. Revista Iberoamericana de Economía Ecológica. 15-28.

Perles Ribes, J. F. (2004). La ventaja competitiva de los destinos turísticos Perles Ribes, J. F. (2004). La ventaja competitiva de Ios

residenciales de la comunidad valenciana. . Papers, 31-39.

Porter, M. (2002). Ventaja Competitiva. Creación y sostenimiento de un de sempeño superior. México D.F.: Grupo Patria Cultural, S.A. de C.V.

Porter, M. (2003). Ser competitivo. Barcelona: Planeta De Agostini Profesional y Formación, S.L.

Porter, M. (2007). Estrategia competitiva. Técnicas para el análisis de los sectores industriales y de la competencia. Mexico, D.F.: Grupo Editorial Patria, S.A. de C.V.

Ros García, J., \& Ros García, M. d. (2004). Información de las fases para la planificación de la rentabilidad empresarial. Revista General de Información y la planificación de la rent.

Quintero, J., \& Sánchez, J. (2006). La cadena de valor: Una herramienta del Quintero, J., \& Sánchez, J. (2006). La
Samiento estratégico. Telos, 377-389.

Sánchez, A., Romero, E., \& Zúñiga, R. (2011). Proyecto Mejorando la Competitividad del Cluster de Software en Venezuela: Diagnóstico y Benchmarking. Caracas: Instituto Centroamericano de Administración de Empresas.

Viñas , M. (2010). La cadena de valor como herramienta estratégica para las Pymes exportadoras colombianas. Dimens, 65-70. 